\title{
DERECHOS HUMANOS, INDIVIDUALISMO Y ESCEPTICISMO
}

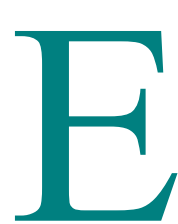

1 lenguaje de los derechos es el lenguaje que predomina en el discurso moral internacional. Los derechos son, por ejemplo, la lengua franca moral de la ONU. ¿Qué quiere decir que todos los seres humanos tienen determinados derechos, como los enumerados en la Declaración Universal de Derechos Humanos?

En este contexto, decir que un individuo tiene un derecho supone afirmar que tiene la pretensión legítima de que tal derecho se le asegure, normalmente ven, primera instancia, por su propio Estado. Sin embargo, si algo se proclama como derecho humano, implica que la comunidad internacional tiene interés en que tal derecho sea respetado. Sin embargo en la práctica esto no ha ido por lo general más allá de una presión moral, sin duda en virtud de la norma internacional que reconoce la soberanía de cada Estado en sus asuntos internos. (La resistencia del Secretario General de las Naciones Unidas a autorizar la intervención en Irak para proteger a la población kurda, constituye un ejemplo actual de este aspecto).

He sugerido que adscribir un derecho a alguien supone afirmar que esa persona tiene la pretensión legítima de que se le asegure tal derecho. Pero la mera declaración de un derecho no explica su fuente. ¿Cómo podemos pues justificar existencia de derechos humanos universales? Esta es la cuestión que me gustaría tratar aquí.

Estoy pensando en esos derechos que figuran virtualmente en cualquier elenco de derechos humanos: libertad de expresión y de religión; un gobierno sometido al principio de legalidad y a la aplicación imparcial de la ley, que no deje lugar a prácticas tales como la tortura o los escuadrones de la muerte; y el derecho a una Constitución que garantice la soberanía popular en una organización política libre. 
Desde una perspectiva estrictamente política tal vez podría argüirse que no interesa llevar el problema de la fundamentación demasiado lejos. Si muchos gobiernos de diversa índole están de acuerdo en suscribir una lista como la de la Declaración Universal de Derechos Humanos, ¿no deberíamos estar ya contentos y dejar potencialmente de lado las arduas cuestiones de su justificación? A mi entender esta propuesta no es satisfactoria por dos razones. Una es que en la práctica, aunque hay muchos gobiernos que firman oficialmente los convenios que recogen los términos de la Declaración Universal de Derechos Humanos, dichos gobiernos violan luego rutinariamente los derechos de sus propios ciudadanos. Esto indica que asumir de boquilla los derechos humanos mediante la firma de algún documento internacional no tiene de por sí gran trascendencia. Dejando aparte consideraciones de orden práctico, la segunda razón es que verdaderamente nos gustaría contar con alguna justificación genérica para los derechos humanos que superara el escrutinio crítico. Si nos conformamos con que los gobiernos suscriban oficialmente los derechos humanos, lo que hacemos es reconocer que los bases de los derechos humanos son puramente convencionales. Esto no nos proporciona ninguna razón para oponernos a la adopción por el mismo gobierno de cualquier otro tipo de convenio en el futuro. Me parece que cualquier teoría satisfactoria de los derechos humanos deberá implicar que los Estados deben respetarlos, con independencia de que sean o no objeto de un convenio internacional.

No obstante la situación actual de los derechos humanos es un tanto irónica. Por un lado vemos que se exige su reconocimiento y puesta en práctica en países como China y Rusia que carecen de una tradición institucional liberal. Por otro, en las democracia liberales, durante los últimos años los filósofos políticos han ido abandonando cada vez más los esfuerzos para apoyar tales demandas, y de hecho han sugerido que cualquier alusión a presuntos principios abstractos es inútil y errónea. Comunitaristas como Michael Walzer y pragmatistas como Richard Rorty han llegado desde diferentes ópticas a conclusiones de orden práctico muy similares a las de conservadores en la línea de Burke como Michael Oakeshott: en vez de apelar a principios abstractos debemos apelar al espíritu de las instituciones consolidadas en nuestra propia sociedad.

Los estudiantes de Pekín o los manifestantes de Moscú recibirían malas noticias si fuera verdad que el único argumento válido fuera el recurso a las prácticas propias de cada sociedad. Las peticiones de reforma podrían zanjarse taxativamente con la respuesta siguiente: «El reconocimiento de los derechos humanos no es la práctica habitual aquí y nunca lo ha sido». Afortunadamente esta teoría relativista es falsa. La tortura, los escuadrones de la muerte o el genocidio nunca pueden 
justificarse diciendo "así es como hacemos aquí las cosas». Si esto es así, por la misma razón su ausencia no puede ya justificarse afirmando «así es como hacemos las cosas por aquí». No me es posible examinar todas las corrientes que han tratado de justificar el carácter universal de los derechos humanos en el espacio de una conferencia. Por tanto, me limitaré a destacar las que me parecen más persuasivas. En primer lugar expondré una de las críticas más consistentes a esta línea de argumentación y luego mostraré como creo que puede hacerse frente a ella.

En 1989, publiqué un libro titulado Theories of Justice, donde defendí la noción de justicia como imparcialidad. No es mi intención volver a trazar todo el proceso que me llevó a extraer tal conclusión. (Tan sólo advertirles que la edición de bolsillo de Theories of Justice saldrá el próximo mes). Trataré de exponer con brevedad la idea básica que se esconde tras la justicia como imparcialidad para decir luego algo más sobre la conexión entre la justicia como imparcialidad y los derechos humanos.

Tomamos como punto de partida la proposición de que las diferentes personas tienen diferentes concepciones acerca de lo que es el bien. Un ejemplo secular nos viene dado por la ética ecológica que concede valor a la integridad de los ecosistemas, una ética sobre el bienestar de los animales que atribuye valor a los estados conscientes de todos los seres que sienten, y la conocida ética utilitarista que asigna valor a la felicidad humana, al placer o a la satisfacción de sus deseos. También hay una amplia variedad de doctrinas religiosas que entrañan una serie de implicaciones conflictivas sobre la forma en que los seres humanos deberían vivir. Además, aunque los egoístas puros escasean, también escasea la gente que da menos importancia a sus propios intereses o a los de su familia que a los intereses de los demás. Como David Hume advirtió, esto basta para generar conflicto. De manera que, incluso si dos personas comparten una misma concepción del bien, esto no conduce necesariamente a un acuerdo en la práctica si valoran el bien de forma diferente según de quien se trate.

Frente a este conflicto, propongo que la gente ceda en sus concepciones sobre el bien y busque una base justa para vivir junto a otros que tienen una concepción diferente. ¿Cómo determinamos esa base justa para una vida en común? La idea que subyace tras la justicia como imparcialidad es que podemos encontrarla preguntando qué principios podrían integrar las bases para un acuerdo bajo unas condiciones hipotéticas para instar a las partes a que alcancen un acuerdo sólo en términos de justicia. 
Desde luego, el ejemplo más conocido de una teoría que refleje esta idea es la elaborada por Jonh Rawls en su libro A Theory of Justice (1971). Según la versión de Rawls (que él denomina «justicia como equidad»), la elección de los principios se realiza tras un velo de ignorancia que impide a la partes conocer determinados aspectos de sí mismas que de otra forma les llevarían a adoptar una solución injusta. Entre las cosas que desconocen se encuentran sus talentos naturales y su posición social, pero también su noción del bien. $\mathrm{Al}$ estar sometidas a esta restricción de información, las partes han de perfilar su concepción de lo bueno cuanto sea posible. No obstante la importancia de restricción estriba en que no pueden perseguir su propia concepción del bien por que no saben cuál es. De manera que están obligados a buscar los principios que aceptarían en la vida real cualquiera que fuera su noción del bien cuando se levantara el velo de ignorancia.

Esta no es la única forma de construir una situación hipotética de elección y en mi libro Theories of Justice considero que no es la mejor. Pero tratar aquí de formulaciones alternativas de justicia como imparcialidad me alejaría mucho del propósito de esta intervención. Procederé pues a examinar la relación existente entre la justicia como imparcialidad y los derechos humanos universales.

Como es bien sabido, Rawls sostiene que de su modelo resultarían unos derechos liberales standard, parecidos a los que mencioné al principio. De esta forma, si tuviéramos que elegir principios ignorando nuestra concepción de lo bueno, parece lógico que no quisiéramos situar ninguna concepción particular del bien en un lugar singularmente privilegiado, puesto que ello podría suponer que luego nos encontrásemos en desventaja. No sería razonable desperdiciar la ocasión de que nuestra concepción del bien acabe por coincidir con la que ha alcanzado una posición privilegiada. Según Rawls todo ello en relación con la situación de igualdad de las partes en la posición original conduciría a un resultado liberal, lo que significa que nadie tiene motivos para no aceptar por lo menos una cantidad igual de derechos civiles o políticos.

Si en algo coinciden los filósofos políticos es en afirmar que la derivación de los principios de justicia desde la posición original está lejos de ser incontrovertible. Creo que es posible señalar sus defectos y que puede formularse una posición original que produzca unas consecuencias más amplias que las de Rawls. Voy a suponer, sin más argumentación, que los derechos humanos pueden justificarse sobre la base de una teoría de la justicia como imparcialidad. 
A partir de ahora, me gustaría recordar una objeción a la justicia como imparcialidad que ha sido ya señalada por otros. En primer lugar, comentaré el perfil que adopta en el reciente libro de Alasdair MacIntyre Whose Justice? Wich Rationality? Según esta objeción, la justicia como imparcialidad es un fraude pues no puede satisfacer sus ansias de estar más allá de toda polémica. Lejos de mediar entre concepciones antagónicas del bien, cuenta con una concepción del bien propia.

Afirma MacIntyre: «el proyecto de una sociedad moderna, liberal e individualista consiste en proporcionar un marco político, jurídico y económico donde sobre la base de un mismo elenco de principios racionalmente fundados, aquellos que sostienen concepciones del bien diferentes e incompatibles puedan convivir pacíficamente en una misma sociedad, disfrutar del mismo status político e intervenir en las mismas relaciones económicas» (pp. 335-336).

MacIntyre procede a señalar las consecuencias que a su juicio se derivan de los principios de una justicia liberal individualista, en los siguientes términos:

«Cada individuo debe tener la misma libertad para proponer y vivir de acuerdo con cualquier concepción del bien que le guste... a no ser que tal concepción del bien suponga una reorganización de la vida del resto de la comunidad según sus términos. Cualquier concepción del bien humano según la cual, por ejemplo, el gobierno tuviera el deber de educar moralmente a los miembros de la comunidad para que adecuaran su vida a tal concepción del bien, podría admitirse hasta cierto punto como una teoría privada para individuos o grupos, pero cualquier intento serio de incorporarla a la vida pública estaría proscrito» (p. 336)

A partir de aquí MacIntyre extrae una conclusión que me gustaría destacar:

«este requisito (en la búsqueda de una concepción del bien) supone no sólo que el individualismo liberal tiene realmente una concepción propia y amplia del bien, que trata de imponer en los ámbitos político, jurídico, social, cultural y allí donde pueda, sino también que al hacerlo su tolerancia hacia concepciones opuestas del bien en la arena pública está seriamente limitada» (p. 336)

Resumiendo, MacIntyre escribe:

«Por tanto, aunque el liberalismo en principio rechaza las pretensiones de cualquier teoría acerca del bien con pretensiones de hegemonía, de hecho es una teoría de este tipo. Además, el liberalismo no ofrece argumentos convincentes en favor de su concepción del bien excepto cuando recurre a premisas que en su conjunto presuponen dicha teoría. Los puntos de partida de los teóricos liberales nunca son neutrales 
respecto a las concepciones del bien humano; son siempre puntos de partida liberales» (p. $345)$.

¿En qué consiste exactamente esta concepción del bien? La primera respuesta que él mismo sugiere es que la concepción individualista del bien humano concede un alto valor a la autonomía de la persona. Según esta idea, la autodeterminación tiene una importancia primordial en la vida del hombre: la gente debería poder decidir cómo vivir y qué pensar, poder manifestar sus creencias libremente y actuar según su propio plan de vida, estar sometida a normas que reconocieran sus derechos a expresarse y actuar garantizando a los demás su capacidad para hacer lo mismo.

Como concepción del bien, obviamente se distingue de la mayoría (aunque no de todas, como veremos enseguida) porque no presupone ninguna visión sustantiva sobre lo que la gente debería creer ni sobre los fines que debería perseguir. Podemos llamarla concepción del bien de segundo orden. Sin embargo, esto no supone que no sea una teoría peculiar del bien. Esto es así desde el momento en que el valor de la autonomía puede ser rechazado. Así, como ha observado William Galston «los fundamentalistas religiosos» podrían negarse a «admitir la capacidad para elaborar y revisar una concepción del bien como bien absoluto, sin interferir en un orden más elevado de intereses de los seres humanos. Podrían declarar que la mejor vida humana requiere la capacidad de recibir un bien externo (la verdad divina) más que formar su propia concepción del bien por sí misma, y aferrarse a esa verdad una vez revelada sin comprobarla». («Pluralism and Social Unity», Ethics 99 (1989) 711-26, p. 714).

Es casi seguro que el sistema liberal de derechos se genera a partir de la concepción del bien como autonomía. Pero parece igualmente cierto que MacIntyre tendría razón, si esta fuera la base donde tuviera que apoyarse la justificación de los derechos liberales. Porque, tal como él afirma, habría ya una concepción del bien que se preferiría sobre las demás, incumpliéndose así las exigencias propias de la justicia como imparcialidad. Se trata pues de saber si hay alguna forma de reinstaurar la justicia como imparcialidad y derivar de ella una serie de derechos humanos similares a los que antes mencioné. Demostraré que podemos. Pero antes, me gustaría volver sobre una visión alternativa de la concepción individualista del bien típicamente liberal.

Pero antes, permítanme añadir que ni siquiera los entusiastas más fanáticos del valor de la autonomía hacen de él el único valor. Críticos poco amables han retratado al liberal ideal como un individuo que pasa todo el tiempo reconsiderando sus creencias y reformulando su concepción del bien sin dejarle casi tiempo libre para vivir. Está claro que esto 
no es lo que buscan los partidarios de la autonomía. Más bien, lo que valoran es vivir de acuerdo con una serie de creencias que han superado el escrutinio, y una concepción del bien que se ha elegido o adoptado después de considerar todas las alternativas. Con todo, se podría decir que esta perspectiva valora la satisfacción de deseos, pero no la satisfacción de cualquier deseo sino sólo la de aquellos que tienen el pedigree adecuado. El origen autónomo de los deseos infunde valor a su satisfacción, por así decirlo.

Sería natural objetar que, si querernos evitar la propuesta de concepciones del bien controvertidas, deberíamos dejar de insistir en que los deseos deben tener el pedigree adecuado que se concretaría en una concepción del bien como autonomía. En cambio, deberíamos adoptar como criterio que cualquier satisfacción de deseos es igualmente buena con independencia de su origen. No obstante, esta sigue siendo una concepción de lo bueno, precisamente la que MacIntyre identifica con el individualismo liberal. Así, cuando afirma (en el fragmento antes citado) que «el individualismo liberal tiene realmente su propia concepción amplia del bien» y que «su tolerancia hacia concepciones opuestas del bien en la arena pública está seriamente limitada», continúa diciendo que esas concepciones antagónicas pueden admitirse en la arena pública sólo bajo la forma de expresiones de preferencias (véase p. 336).

Como ocurre con la autonomía, esta visión de la concepción liberal individualista del bien constituye una concepción de segundo orden. No dice lo que es bueno de hecho, pero afirma que es bueno que la gente tenga éxito en su búsqueda del bien tal como ella misma lo conciba. Esta nota del individualismo liberal a la MacIntyre explica (lo que me parece que no explica MacIntyre) como puede afirmar a la vez que el individualismo tiene una concepción propia del bien y que «el liberal se compromete a que no haya ningún bien predominante» (p. 337). Esta paradoja se resuelve si asumimos que el individualismo liberal no tiene una concepción del bien de primer orden sino que transforma todas las concepciones del bien de primer orden en preferencias y sostiene que el bien consiste en la satisfacción de preferencias.

Queda claro que se trata de una teoría coherente sobre el bien. Realmente es una variante de la teoría utilitarista, la variación estriba en que aquí el bien consiste en la satisfacción de deseos más que en la de, digamos, placer. Hay que reconocer que las conclusiones liberales no se extraen de premisas utilitaristas de forma tan automática como en el caso de la concepción del bien como autonomía. Sin embargo, si presentamos una ordenación de preferencias donde tener la posibilidad de hacer lo que uno quiera sea más importante que tener la posibilidad de impedir al resto que haga lo que quiera, las implicaciones liberales se vuelven muy plausibles. Supongamos que hasta cierto punto 
MacIntyre tiene razón al pensar que el utilitarismo conduce a la aprobación de las instituciones liberales. Pero, ¿acaso pueden sostenerse tales instituciones sin que se invoque una noción específica del bien? ¿Podemos hacerlo aun sin concebir el bien como autonomía o satisfacción de deseos y luego derivar prescripciones liberales tales como las que figuran en las listas standard de derechos humanos?

Me gustaría añadir que hay un argumento a favor de las conclusiones liberales que no apela a una teoría específica del bien sino a una epistemología específica, especialmente a una que ve con escepticismo la posibilidad de establecer la superioridad de cualquier concepción del bien con la certeza suficiente como para justificar que se le de una posición privilegiada. El argumento es el siguiente: dado que no hay esperanza de alcanzar un acuerdo racional sobre ninguna concepción del bien, debemos buscar un acuerdo cuyas bases no presupongan ninguna concepción particular del bien, y la justicia como imparcialidad nos ofrece la mejor base disponible.

No descubro nada nuevo al sugerir que hay una conexión íntima entre liberalismo (entendido aquí como una doctrina política tolerante, etc.) y escepticismo. Al contrario, creo que siempre ha sido así y que siempre se ha admitido. La opinión liberal al respecto procede de una concepción de racionalidad que atendió a los avances de la ciencia desde el siglo XVII y de una determinada respuesta a las guerras de religión durante el mismo periodo. Cualquiera que se interese por las raíces históricas de las tradiciones, deberá admitir que MacIntyre resulta asombrosamente miope en su tratamiento del liberalismo. Cualquiera que no supiera nada sobre el asunto y lo conociera a través de MacIntyre saldría con la impresión de que el liberalismo fue simplemente arrojado a la existencia por algo llamado la ilustración». En la medida en que puede decirse que tuvo alguna fuerza motriz, ello constituye lo que MacIntyre llama «el proyecto de la ilustración»: el deseo de deducir la moral de la pura noción de racionalidad, en lugar de tal o cual concepción de la racionalidad históricamente condicionada.

Hay dos cosas equivocadas en esta historia. En primer lugar, está claro y siempre lo ha estado que el liberalismo tiene su propia concepción de la racionalidad, que difiere, por ejemplo, de la de Aristóteles o Aquino. El ascenso del liberalismo coincidió con el progreso de las ciencias físicas en su forma moderna, y no es casual que Newton llegara a ser virtualmente el patrón de los liberales. La justicia como imparcialidad, que debe entenderse como una reelaboración contemporánea de algunas ideas liberales tradicionales, está también ligada a una concepción característica de la racionalidad moderna, IV., pero hay muy buenas razones para sumarse a esta concepción, basada en el éxito efectivo de la ciencia en la predicción y el control de la naturaleza. 
Por ejemplo, el método que empleó Santo Tomás de Aquino, que es el filósofo favorito de MacIntyre, para encontrar la respuesta a una determinada cuestión consistía en recopilar lo que las autoridades (especialmente Aristóteles) habían dicho sobre el particular, situarlas en un esquema preestablecido, y luego decidir a la luz de la razón qué respuesta elegir. Parece que nunca se le ocurrió que la mejor forma de encontrar la respuesta a muchas preguntas consiste en buscar por uno mismo, o en tratar de alcanzar la máxima satisfacción de uno mismo en lugar de apelar a los que creemos que así hicieron. Está claro que se trata de una concepción de la racionalidad distinta a la que era común entre los que recibieron la influencia de los métodos propios de la ciencia post-galileana. A mi entender se trata además de una concepción indudablemente inferior.

El segundo error en el retrato que hace MacIntyre de los orígenes del liberalismo es que ignora por completo que sus raíces se encuentran en los conflictos religiosos que acompañaron y siguieron a la Reforma. La defensa de la libertad religiosa resultó de la intransigencia de los conflictos entre católicos y protestantes. Resultó evidente para ambos lados que no se resolverían tratando de convertir a los miembros del otro lado apelando a argumentos racionales, sino estableciendo la armonía religiosa. Incluso penas civiles, políticas, económicas, y en casos extremos físicas, legalmente establecidas se revelaron incapaces de producir un cambio de creencias auténtico y duradero. Ni las expulsiones masivas, ni los genocidios gozaron de ninguna eficacia para restaurar la ortodoxia en una sociedad donde las diferencias religiosas habían ido arraigando. La libertad religiosa (que al principio se extendió sólo a los cristianos o a los que creían en un dios descrito de alguna manera) se reclamó como una respuesta práctica humana a esta situación.

Por supuesto, no debemos admitir que la experiencia europea durante los siglos XVI y XVII sea representativa. Los intereses en juego durante el conflicto que rodeó la Reforma y la Contrarreforma, estaban al fin y al cabo en gran medida relacionados con aspectos de organización social y especialmente con la situación de la autoridad religiosa. Que tales disputas fueran inmunes a la persuasión racional no demuestra necesariamente que otras cuestiones más profundas relacionadas con las creencias religiosas no puedan resolverse de esta forma. Si ampliamos el alcance de nuestro análisis encontraremos realmente gran cantidad de conversiones religiosas voluntarias en la historia. No obstante sería complejo establecer algún modelo que indicara la dirección hacia donde tienden tales conversiones. 
Puestos a determinar un modelo, se me ocurre que hay uno donde la gente cambia de religión por dos tipos de motivos que son ajenos a la falsedad o verdad intrínseca ya de la religión que dejan o de la religión que adoptan. Uno consiste en el deseo de progreso social individual, de ventajas económicas o de emancipación política. Un ejemplo es la conversión de muchos judíos al cristianismo en Alemania y en Austria durante los siglos XVIII y XIX. Otro ejemplo más trivial está representado por el fenómeno americano de «intercambiar» denominaciones (v.gr. de baptista a presbiteriano o episcopaliano) paralelo a las aspiraciones de ascender de status. La otra razón para convertirse, que es básicamente la misma pero que puede servir a gran escala, es el deseo de asociarse con una cultura más sofisticada o de alguna manera más poderosa, y tomar la religión como una forma relativamente sencilla de hacerlo mucho más fácil por ejemplo, que dominar su literatura o su ciencia. Tanto el islam en épocas tardías como la cristiandad en épocas más recientes, se han beneficiado de esta tendencia propia de gente relativamente «atrasada».

Debo reconocer que todo esto es muy superficial. Sin embargo, la fuerza arrolladora de las pruebas a favor del escepticismo resulta aplastante. Es difícil no impresionarse al advertir que tanta gente haya dedicado tanto esfuerzo durante tantos siglos a un asunto de la mayor trascendencia con tan poco éxito en el modo de asegurar una convicción racional entre los que no estaban inicialmente predispuestos a favor de sus conclusiones.

Supongo que, si la defensa del escepticismo puede funcionar respecto de las concepciones del bien basadas en la religión, también servirá a fortiori para otras concepciones. Por poner un ejemplo personal, me siento fuertemente atraído por una ética ecocéntrica y apoyaría que se sacrificara la satisfacción de una cantidad considerable de deseos humanos (especialmente si el sacrificio estuviera equitativamente distribuido) en la búsqueda de una concepción ecológica del bien. Creo que pueden aportarse argumentos mostrando lo más claramente posible las consecuencias que se derivan de seguirla o no y apelando a un tipo de sentimiento que al fin y al cabo sería, creo yo, de la misma naturaleza que el que fundamenta muchas religiones. Pero sigo sin ver cómo sus pretensiones pueden presentarse de tal forma que demuestren que no sería razonable adoptar un punto de vista distinto, y estimo que cualquier otra concepción del bien está igualmente sujeta al mismo inconveniente.

Me gustaría terminar mi exposición hablando de los últimos escritos de John Rawls. He dicho antes que la versión rawlsiana de la justicia como imparcialidad es con mucho la más conocida. Y verdaderamente, desde A Theory of Justice hasta sus escritos más recientes, Rawls ha 
afirmado que «el liberalismo es una doctrina política que reconoce que hay muchas concepciones del bien conflictivas e inconmensurables, siendo cada una de ellas compatible con la plena racionalidad de la persona humana», a partir de esto ha sacado la conclusión que la prioridad de lo justo (right) sobre lo bueno (good) «es característica del liberalismo como doctrina política» en el sentido que «una estructura básica justa y las instituciones de su entorno establecen el marco dentro del cual pueden darse las concepciones (del bien) admisibles» (John Rawls, «Justice as Fairness: Political not Metaphysical», Philosophy and Public Affairs 14 (1985) pp. 223-51, cit. p. 248). De aquí se desprende a mi entender como consecuencia lógica, que estamos equivocados si nuestra concepción del bien (una doctrina religiosa por ejemplo) lleva consigo la idea de que es lo suficientemente segura como para justificar que se imponga a quienes la rechazan. Entendida de esta forma, la justicia como imparcialidad puede sostenerse como la teoría idónea para tratar con concepciones antagónicas del bien, cualquiera que sea su forma.

Sin embargo en el artículo que he citado, que data de 1985, Rawls declaraba que el fundamento de la justicia como imparcialidad no consistía en que fuera la forma correcta para tratar la diversidad. Antes bien, era la que aportaba las mejores posibilidades de alcanzar un acuerdo bajo las condiciones de las sociedades democráticas liberales contemporáneas. Obviamente la dificultad estriba en que el deseo generalizado de llegar a un acuerdo no proporciona una razón para alcanzarlo sobre unos determinados términos. MacIntyre podría haber indicado que si (como él noes exhorta) todos aceptaran el tomismo entonces (tautológicamente) el acuerdo se habría alcanzado. Si se persuade a la gente de que el acuerdo se alcanzará en los términos que ofrece la justicia como imparcialidad, tenemos que darles alguna buena razón para pensar que esos son los términos correctos. Y vuelvo a afirmar que el escepticismo, tal y como lo he definido, es el único buen argumento disponible.

Parece que el mismo Rawls reconoce el problema, y le da su propia respuesta, pero ésta parece muy poco prometedora. Según él, las pretensiones de la justicia como imparcialidad dependen de que forme en parte de una «consenso por superposición» (overlappping consensus). «Suponemos que esas ideas (vgr. la «justicia como imparcialidad» de Rawls) son susceptibles de ser ratificadas por cada una de las doctrinas morales globales y antagónicas que influyen en una sociedad democrática razonablemente justa» (p. 246). Rawls señala tres doctrinas globales que podrían respaldar la concepción de la justicia como imparcialidad tal como él la entiende: una visión religiosa que incorpore el principio de la tolerancia, una «concepción liberal de la moral que 
sea global como la de Kant y Mill» y por último una concepción de la «justicia como imparcialidad que no resulte de una doctrina más amplia sino que sea por sí misma suficiente para expresar valores que normalmente pesan más que cualesquiera otros valores que se le enfrenten, al menos bajo condiciones razonablemente favorables» (p. 250). (Presumiblemente la tercera debe entenderse como una teoría axiológica pluralista donde la equidad deberá transigir con otros valores sustantivos).

Pueden hacerse dos objeciones a esta idea. La primera es que, como fórmula para «vender» la justicia como imparcialidad (así es como la presenta Rawls), resulta bastante ineficaz, incluso en las sociedades democráticas liberales contemporáneas. Por supuesto, hay un número de doctrinas religiosas que incluyen la tolerancia como parte de su concepción del bien, y seguramente unas cuantas han llegado a atenuarse tanto como para incorporar toda la gama de la justicia como imparcialidad. Pero muchas no. Si la justicia como imparcialidad ha de apelar a las creencias genuinas de la gente en tales sociedades, no haremos grandes progresos con tomistas a la MacIntyre, la mayoría del los baptistas del sur en USA, muchos musulmanes en Europa del Este y demás. Una teoría de la justicia que se complica y muere cuando se la enfrenta con huesos duros de roer como estos, no es de mucha utilidad como propuesta práctica desde el momento en que deja de funcionar cuando más se la necesita.

La segunda, y más importante, objeción es que el proyecto que representa la justicia como imparcialidad se trivializa necesariamente por esta forma de entenderlo. Se supone que la idea global de la justicia como imparcialidad consistía en que ofrecía el modo de arbitrar concepciones antagónicas del bien incluyendo, por supuesto, las concepciones no liberales del bien. Según la interpretación de Rawls, sólo resuelve los casos donde las concepciones del bien ya incluyen la justicia como imparcialidad como parte de un «consenso por superposición» (overlapping consensus).

Rawls nos dice «ese consenso por superposición (sobre la justicia como imparcialidad) resulta mucho más estable que otro fundado sobre criterios que manifiesten escepticismo e indiferencia hacia valores religiosos, filosóficos o morales, o que contemplen la aceptación de principios de justicia meramente como un modus vivendi prudente según del equilibrio de fuerzas sociales existente» (p. 250). El segundo punto se acepta con facilidad toda vez que la estabilidad de los principios de justicia basada en un modus viviendi depende de la conversación del equilibrio de fuerzas que lo sustentan. Respecto al primero, parece que Rawls distorsiona y rechaza el único fundamento de la justicia como imparcialidad que tiene alguna fuerza independiente. Concluye 
erróneamente que la nota más relevante del escepticismo es «su indiferencia hacia valores religiosos, filosóficos y morales» en lugar de las dudas sobre la certeza con la que alguno de ellos podría mantenerse justificadamente. $Y$ entonces condena el escepticismo por ser una garantía de estabilidad más débil que el consenso por superposición. Por supuesto que lo es: si se da un consenso por superposición, entonces por definición la gente ya ha aceptado la teoría de la justicia como imparcialidad. Decir que permanece estable bajo tales condiciones es decir poco más que si la gente cree en algo entonces ya estará dispuesta a aceptar una teoría que le diga la misma cosa. El quid del escepticismo, tal como yo lo entiendo, es que proporciona una base para defender la teoría de la justicia como imparcialidad apropiada para la situación de la gente cuyas concepciones del bien no la contienen.

(Traducción de Mercedes Carreras) 
